\title{
Hydrocarbon recovery by condensed rotational separation
}

\author{
H. P. van Kemenade • J. J. H. Brouwers
}

Received: 5 July 2011/ Accepted: 26 January 2012 / Published online: 14 February 2012

(c) The Author(s) 2012. This article is published with open access at Springerlink.com

\begin{abstract}
Condensed rotational separation (CRS) is a novel method of gas-gas separation. It is based on pressure distillation at semi-cryogenic temperatures whereby one of the components is condensed, thereby forming a mist of micron-sized droplets. These are separated by applying the rotational particle separator (RPS). The RPS is a compact device for separating micron-sized particles from gases by centrifugation. In this paper, CRS is presented as an efficient method to upgrade natural gas fields contaminated with $\mathrm{H}_{2} \mathrm{~S} / \mathrm{CO}_{2}$. The process consists of two pressure reduction steps. The first step results in maximizing the methane concentration of the gas. The pressure of the separated liquid is further reduced to maximize the concentration of liquid $\mathrm{H}_{2} \mathrm{~S} / \mathrm{CO}_{2}$ whereby the released gas is re-fed to the inlet of the first step. Basic principles, process lay-outs and equipment are discussed. Compared to conventional processes, CRS excels in compactness and minimum usage of energy.
\end{abstract}

Keywords Hydrocarbon recovery · Sour gas · Condensed rotational separation

\section{Introduction}

Separation of gas mixtures is an issue in various energyrelated processes. Examples are the separation of $\mathrm{H}_{2} \mathrm{~S}$ and $\mathrm{CO}_{2}$ from methane to upgrade sour gas fields and the

H. P. van Kemenade $(\bowtie)$ · J. J. H. Brouwers

Eindhoven University of Technology, PO box 513, 5600 MB,

Eindhven, The Netherlands

e-mail: h.p.v.kemenade@tue.nl

J. J. H. Brouwers

e-mail: j.j.h.brouwers@tue.nl capture of $\mathrm{CO}_{2}$ from coal fired power plants to reduce the emissions of greenhouse gases. The mature industrial method for these applications has been the amine absorption technology. The contaminated gas is bubbled through solvent in an absorber column where the solvent absorbs the contaminant. Afterwards, the solvent is regenerated by heating and/or flashing to a lower pressure. The process becomes increasingly uneconomical at higher contamination levels due to the intensive energy requirements and large equipment size (Olajire 2010). An alternative method is to separate on the basis of phase separation. The mixture is cooled to semi-cryogenic temperatures, whereby the contaminant condenses partially and after which the condensate is separated. Several processes have been developed on this principle, for example the Total Sprex process to remove $\mathrm{H}_{2} \mathrm{~S}$ from natural gas (Mougin et al. 2008), Cryocell by CoolEnergy to remove $\mathrm{CO}_{2}$ from natural gas (Hart and Gnanedran 2009), Controlled Freeze Zone by Exxon for sour gas fields (Burgers et al. 2011) and the Alstom anti-sublimation process (Clodic et al. 2005). The energy consumption of these processes is limited and makes them useful for high contaminant levels. The drawback is the large size of the installations and capital costs involved.

Instead of fractional distillation employing temperature differences, one can separate by flash evaporation or pressure distillation. The advantage is simplicity and short residence time, i.e. small equipment and limited investment costs. A technological challenge is, however, posed by the feature that rapid cooling of binary or multi component mixtures of gases to temperatures where one, or some of the components preferentially condense, leads to a mist of very small droplets with diameters of 1-10 $\mu \mathrm{m}$ (Schaber et al. 2002). The phenomenon is known to occur by aerosol formation in flue gases of biomass combustion installations 
(de Best et al. 2008), condensate droplets resulting from cooling of wet natural gas (Austrheim 2006) and has also been measured in experiments with $\mathrm{CH}_{4} / \mathrm{CO}_{2}$ mixtures (Willems et al. 2010a). As the micron-sized droplets are difficult to separate from the gas stream (Hinds 1999), the size advantage of pressure distillation is often lost in the required separator. For a process which relies on fast phase change as a means of separation to be economical and practical, it is necessary to have a device capable of capturing micron-sized droplets with high collection efficiency at low pressure drop and a small footprint.

Wire mesh, vane type and cyclonic separators are standard as mist extractors for the small liquid droplets that are not separated by gravity in the main body of the separator (Gas Processors Suppliers Association 2004). These cyclones are used for water and condensate removal but have not been applied for removing condensed contaminants, such as $\mathrm{CO}_{2}$ or $\mathrm{H}_{2} \mathrm{~S}$. This is because cyclones can only handle condensing droplet sizes above $10 \mu \mathrm{m}$ (Gas Processors Suppliers Association 2004; Clift 1997; Svarovsky 1984). To achieve such droplet, sizes requires unrealistically large droplet growth pipes. It is well known in laboratory chemical applications that microcyclones can separate micron-sized droplets having diameters as small as $1 \mu \mathrm{m}$, but then the flow is very small and orders of magnitude less than the flow in gas well applications. Alternatively, one can improve separation efficiency by increasing swirl velocity to supersonic velocity but at the costs of large pressure drops (Schinkelshoek et al. 2008).

A more feasible solution is provided by the rotational particle separator, abbreviated by RPS (Brouwers et al. 2012). The RPS consists of a cylindrical pipe wherein a rotating element is placed. The rotating element is a simple rotating body consisting of a very large number of axial channels of a few millimeters in diameter. In such a channel, the micron-sized droplets are centrifuged to form a liquid film at the channel-wall, which is ripped of at the exit of the channel in the form of droplets; typically $20 \mu \mathrm{m}$ or larger. These droplets are separated according to the working principles of ordinary axial cyclones. The rotating element can receive its momentum for rotation by prerotation of the gas entering the rotating element, and/or by external drive through an electrical motor which is indirectly connected through a magnetic field.

In the CRS process, the RPS is used to separate the micron-sized droplets. The contaminated gas is rapidly lowered in temperature -50 to $-100^{\circ} \mathrm{C}$ and reduced in pressure (30-40 bar). A mixture forms which consists of predominantly the gaseous phase with a mist of small micron-sized droplets of liquid/solid contaminant. The separation process is further enhanced by introducing a second step. The collected liquid/solid slurry is flashed to a liquid/gas mixture at 20 bar. The liquid is purified, while most of the gas which was dissolved in the liquid/solid slurry evaporates. This gas is re-fed into the gas stream in the first part of the process.

In this paper, we shall address the important aspects of CRS. In "Process thermodynamics", the CRS process is compared to fractional distillation on thermodynamic principles. In "Equipment", the design considerations are applied to a fractionation scheme for sour gas fields. Other potential applications of the CRS technology are shortly discussed in "CRS as bulk sweetening process", "Conclusions" are in the final section.

\section{Process thermodynamics}

In Fig. 1, the phase diagram of the $\mathrm{CH}_{4}-\mathrm{CO}_{2}$ system is shown. The solid-phase boundaries are shown by the dashdotted lines. Also depicted in Fig. 1 is the principle of condensed rotational separation. The incoming mixture with methane concentration $X_{\text {in }}$ is chilled by a combination of cooling and expansion to a point close to, or just over, the solid boundary of the vapour-liquid phase (within $5^{\circ} \mathrm{C}$ ), where the recovery of methane is maximal (van Wissen 2006). This point is indicated by A in Fig. 1. Because of binary condensation, a mist of small droplets is formed with composition $X_{A l}$. The vapour phase has a composition $X_{A v}$ and is separated. The droplets are separated by the RPS to obtain a liquid stream. This liquid stream is subsequently heated and expanded to obtain liquid droplets with composition $X_{B l}$ and a gas with composition $X_{B v}$, at a point $(p, T)$ even closer to the solids boundary. The resulting gas

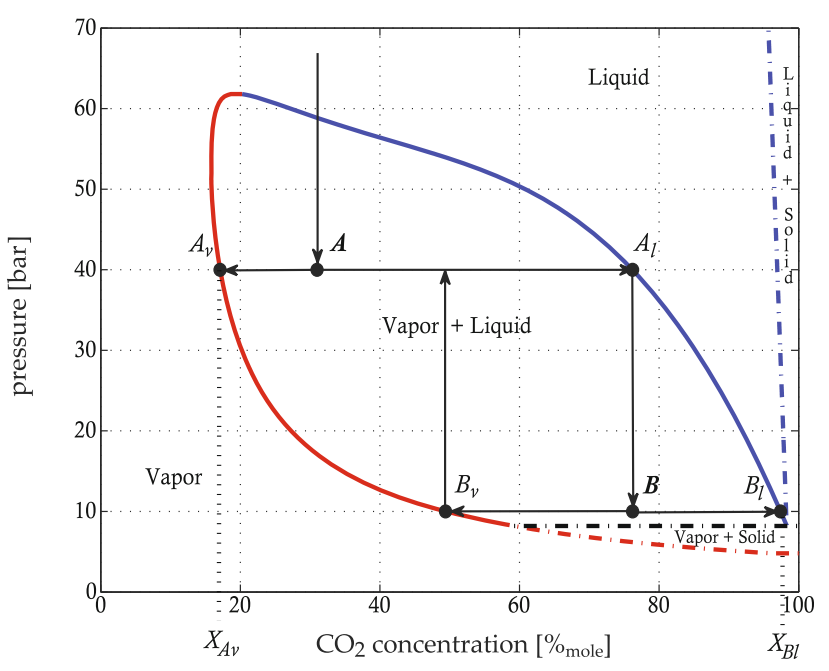

Fig. $1 P-X$ diagram for the $\mathrm{CH}_{4}-\mathrm{CO}_{2}$ system. The incoming gas mixture is cooled and expanded to point $A$ in the phase diagram where it is separated in a gas stream with composition $X_{A v}$ and a liquid stream with composition $X_{A l}$. In the second flash, the liquid is separated into a liquid with compostion $X_{B l}$ and a gas $X_{B v}$. This gas is mixed with the incoming gas on line $A_{v}-A_{l}$ 
has a composition that is not far from the original untreated feed gas and is re-fed to the original gas entering the installation. The process can operate over a wide range of feed gas compositions and produce specification product, both the gas and liquid streams.

In CRS, the cooler serves to bring the mixture to the desired temperature prior to expansion. The expansion and subsequent formation of mist are extremely fast processes and take place in about 10-100 ms (Gas Processors Suppliers Association 2004; Bansal 2012). The total residence time of the gas in CRS is estimated at $0.1-0.2 \mathrm{~s}$. This is about 100 times faster than separation by condensation using adiabatic cooling as occurs during temperature distillation using a demethanizer column. The size advantage of the CRS process particularly pays out in case of high pressure applications where equipment has to be small.

To obtain the same product specification with temperature distillation, the demethanizer column has to be operated between the temperatures $T_{1}$ and $T_{2}$ as shown in Fig. 2. The limiting temperature difference between vapour and liquid for temperature distillation is $\Delta T_{1}$ in Fig. 2. At this temperature difference, the vapour condenses without change in composition. The heat and mass transfer surface in the column has to be dimensioned in such a way that this temperature difference is not exceeded. As the heat exchangers in the CRS process are not used for separation, the full available temperature difference, approximately $\Delta T_{2}$ in Fig. 2, can be used to reduce the size. For an equal design, the size of the heat exchangers in CRS can be $\Delta T_{1} / \Delta T_{2}$ smaller than a distillation column.

In reality, raw natural gas is not a binary mixture, but contains traces of $\mathrm{N}_{2}, \mathrm{C}_{2} \mathrm{H}_{6}$ and $\mathrm{H}_{2}$. The presence of $\mathrm{H}_{2} \mathrm{~S}$ in a $\mathrm{CO}_{2}-\mathrm{CH}_{4}$ system lowers the temperature at which solids are formed. In that case, significantly higher purities of methane in the gas phase can be reached while operating

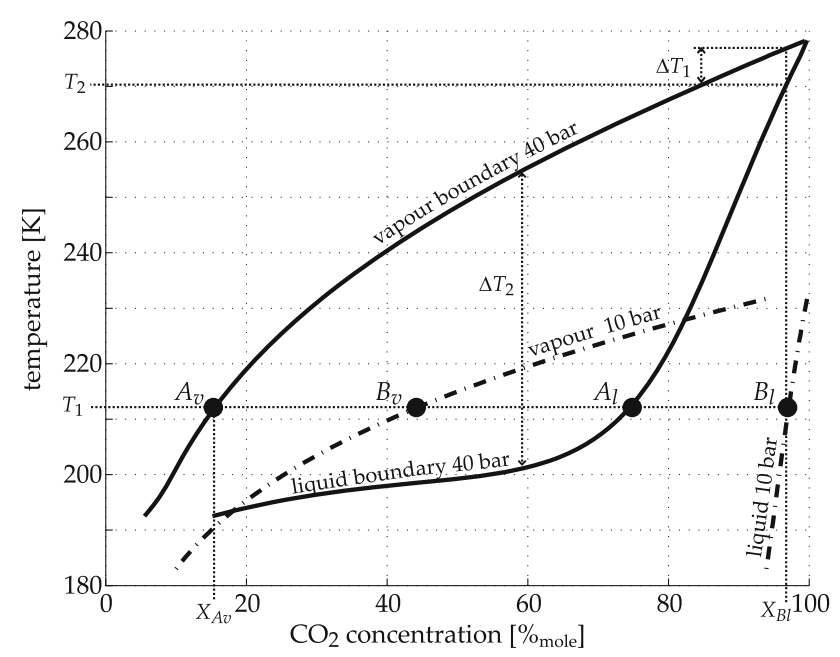

Fig. $2 T-X$ diagram for the $\mathrm{CH}_{4}-\mathrm{CO}_{2}$ system at 40 and 10 bar in the vapor-liquid regime (see also "Equipment"). Furthermore, at temperatures below the solid boundary, multicomponent mixtures have a vapour-liquid-solid region instead of a vapour-solid region. This makes operation of CRS possible in this region, as long as the solid fraction does not become dominant. By operating CRS in the vapour-liquid-solid region, much higher purities of methane on the gas phase can be achieved, compared to operation of CRS in the vapour-liquid region.

\section{Equipment}

With the exception of the rotational particle separator, the equipment for CRS can be sourced from existing processes like hydrocarbon recovery and liquified natural gas production (Gas Processors Suppliers Association 2004; Kidnay et al. 2006). The compressor in the regeneration loop operates at pressure ratio $<4$, implying that a standard single stage compressor can be used. The compressor efficiency is estimated at 0.8 . In this study, a heat to power efficiency of 0.4 is assumed.

For estimating the dimensions of the heat exchangers, a packing of $300 \mathrm{~m}^{2}$ per $1 \mathrm{~m}^{3}$ volume is assumed. This corresponds to the upper limit for a traditional coil wound design and the lower limit for a plate-fin heat exchanger (Linde). The heat and mass transfer coefficients are calculated according to (VDI-Wärmeatlas et al. 2006) with additional data from (Schaber et al. 2002) and (Neeraas et al. 2004a, b). To estimate the size of the heat exchangers, we use the transfer unit approach. The number of (heat) transfer units is defined as $\mathrm{NTU}_{\mathrm{H}}=U A / C_{\text {min }}$ with $U$ being the overall heat transfer coefficient, $A$ the heat transferring surface, and $C_{\text {min }}$ the smallest capacity flow. It can be argued on thermodynamic principles (VDI-Wärmeatlas et al. 2006; Bejan 1997) that a NTU number of 2 in most cases is the optimal value.

The formation of mist during the CRS process is investigated both theoretically and experimentally in a small scale laboratorium set-up using a RPS (Willems et al. 2009; Bansal et al. 2011; Bansal 2012) for $\mathrm{CH}_{4}-\mathrm{CO}_{2}$ and $\mathrm{N}_{2}-\mathrm{CO}_{2}$ mixtures. Droplet sizes down to $1 \mu \mathrm{m}$ were reported and observed. To verify the scale laws for the RPS, a demonstration unit connected to an atmospheric test rig with water and air as working fluids was realised (Willems et al. 2010b). The test setup approximately models a $24 \mathrm{~m} 3 / \mathrm{s}$ (80 MMscf/d) equivalent installation on a natural gas well. The design is suited for large liquid loads and is schematically depicted in Fig. 3.

Often, the performance of a gas scrubber is presented in the form of a sizing or load factor $K$ as used in the Sounders-Brown equation. The required gas scrubber area (footprint) can then be calculated from 


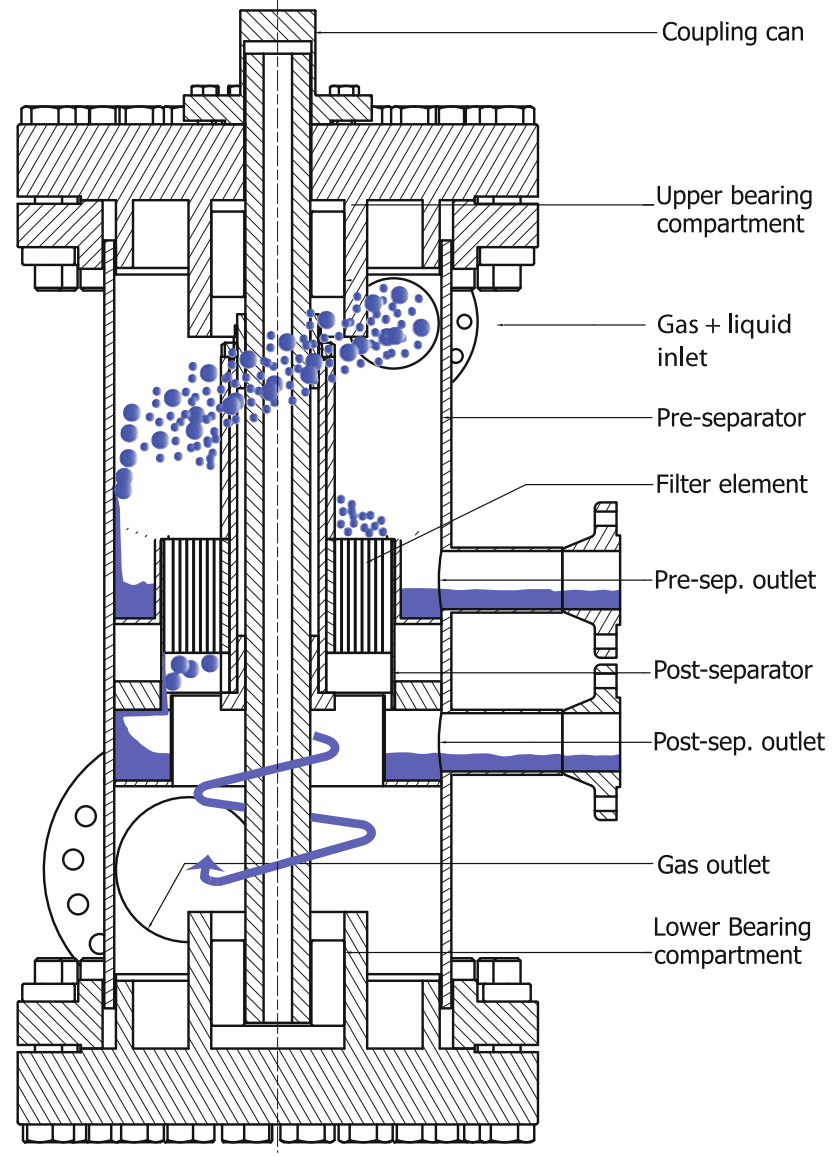

Fig. 3 Schematic drawing of the rotational particle separator

$A=\frac{Q_{A}}{K} \sqrt{\frac{\rho_{l}}{\rho_{l}-\rho_{g}}}$

The load factor $K$ is a direct measure for the required footprint of the installation and has the unit of velocity. In Fig. 4, we have compared the best practice of scrubbers with that of a RPS (Brouwers et al. 2012). Here $d_{p 50}$ is the droplet size that is separated with $50 \%$ probability. Only under atmospheric pressure, a $d_{p 50}$ of $3 \mu \mathrm{m}$ is the footprint of a cyclone deck comparable to that of a RPS. At elevated pressures, the RPS can be an order smaller in size. At all pressures, the RPS is also capable to separate droplets down to $1 \mu \mathrm{m}$ as is necessary in the CRS process.

The natural gas processing will take place at temperatures as low as $-90^{\circ} \mathrm{C}$ which limits the use of several metal alloys. Within the gas, some highly corrosive substances, e.g. chlorides and sulphides, can be present that can lead to several forms of corrosion, e.g.: pitting corrosion, sulphide stress cracking and crevice corrosion. Although the unit is operated in the "severe sour regime", due to the low temperatures the corrosion risk is less severe (Schweitzer 1996). The lab-scale RPS is manufactured from duplex stainless steel (van Wissen 2006) because of the low

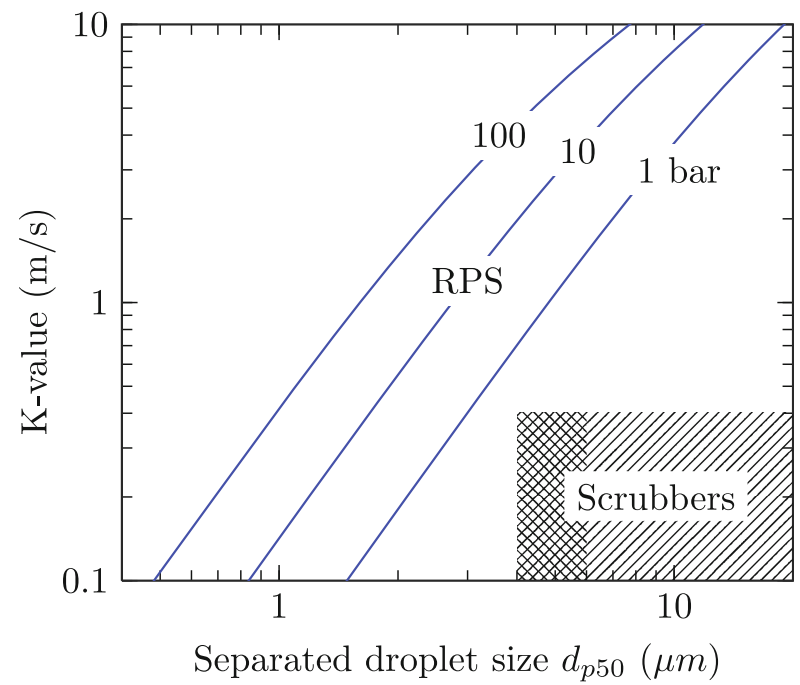

Fig. $4 K$ values for the RPS and scrubbers. Crosshatch pattern indicates best practice using cyclone decks. $d_{p 50}$ is the droplet size that is separated with $50 \%$ probability

thermal expansion coefficient. Duplex steels often get brittle at temperatures below $-70^{\circ} \mathrm{C}$, so from the austenitic family AISI $316(\mathrm{~L}, \mathrm{LT})$ is also considered (Willems et al. 2009).

\section{CRS as bulk sweetening process}

The case study presented in this section is based on the data for the combined stream of the Arabiyah and Hasbah gas fields in Saudi Arabia (Alami 2010). The main components of the gas are $77 \%_{\text {mole }} \mathrm{CH}_{4}, 9 \%_{\text {mole }} \mathrm{N}_{2}, 8 \%_{\text {mole }} \mathrm{CO}_{2}$, $4 \%_{\text {mole }} \mathrm{H}_{2} \mathrm{~S}$ and $1 \%_{\text {mole }} \mathrm{C}_{2} \mathrm{H}_{6}$. Standard technology for gas sweetening is amine treatment such as the sulphinol process (Alami 2010). The sour gas is fed at elevated pressure (70 bar) to an absorbtion tower where it is contacted with the solvent to absorb the acid gas components. The rich solvent is subsequently flashed to a lower pressure to recover most of the absorbed hydrocarbons. The rich solvent is then regenerated by steam heating in the stripper column. As shown below, application of CRS as a bulk sweetening process results in considerable savings on both size and energy demand.

The fluid phase and energy calculations are performed with use of custom written models in the mathematical environment of MatLab. The phase equilibrium model is based on equi-fugacity calculations with use of the PengRobinson Equation of State, Van-der-Waals one-fluid mixing rules and experimentally fitted binary mixture interaction parameters taken from literature. A freeze out model to predict the boundary of solid $\mathrm{CO}_{2}$ formation is also incorporated and is based on the fugacity calculation of a pure solid with use of a pure substance sublimation 
pressure correlation and a constant solid phase molar volume. Isentropic, isenthalpic and isobaric models were constructed to perform the energy calculations. Enthalpies and entropies in these models are calculated with the use of departure functions, ideal gas heat capacities from literature and the ideal gas state as reference state, as described in Reid 1984). The custom written code allows for an easy and flexible optimization of the process. The final results were checked against the computer package Aspen-Plus and the available experimental data (Fig. 5).

The temperature and pressure of the first point of separation ( $A$ in Fig. 6 ) are chosen such that the gas purity in methane and ethane is maximum. It corresponds to a temperature of $-100^{\circ} \mathrm{C}$ and a pressure of 22 bar. This is in the VLS region whereby liquid and solids are of approximately equal amount and form a slurry that can be handled by the RPS. To reach the desired temperature and pressure, the feed gas is first cooled as a single phase in a heat exchanger to $-70^{\circ} \mathrm{C}$ Thereafter, the feed gas is reduced in temperature and pressure through a throttling valve. In the RPS, the gas and the liquid/solid slurry is separated. The slurry is heated to obtain a pure liquid and flashed to a pressure of 4 bar and a temperature of $-70^{\circ} \mathrm{C}$ (point $\mathrm{B}$ in Fig. 6). Gas and liquid are separated by the second RPS and the gas is mixed with the incoming gas stream of the first separator after compression to $22 \mathrm{bar}$ and cooling to $-100^{\circ} \mathrm{C}$. Almost all the methane is recovered in the purified gas (99.9\%) and $84 \%$ of the ethane. In the liquid, $87 \%$ of the $\mathrm{CO}_{2}$ and $87 \%$ of $\mathrm{H}_{2} \mathrm{~S}$ is recovered.

We consider the case that CRS delivers the produced gas at a pressure of 22 bar. In that case, the energy consumption of CRS is due to recompression of the gas after the second flash, hydrocarbons lost in the liquid and the chiller. Although the product streams can be used to cool the incoming gas, an external chiller is necessary to compensate for the heating by the compressor and the limited size of the heat exchangers. The combined energy consumption recalculated to primary energy using a conversion factor of heat to power of 0.4 is $2.2 \times 10^{5} \mathrm{~J}$ per $1 \mathrm{~kg}^{-1}$ of feedstock gas. This corresponds to $0.6 \%$ of the heating value of the incoming hydrocarbons. A breakdown of the energy consumption is given in Table 1 .

The main contribution to the size of the CRS installation is by the heat exchangers. As is usual in cryogenic gas processing, they can be combined in a "cold box". For estimating the size of the heat exchangers, we have assumed, in line with common practice (cf. "Equipment"), a number of transfer units NTU $=2$ and a packing of 300 $\mathrm{m}^{2}$ of heat exchanging surface per $1 \mathrm{~m}^{2}$. The resulting volume of the cold box is $3.8 \times 10^{5} \mathrm{~m}^{3}$ per $1 \mathrm{~kg}^{-1}$ of feedstock gas (see Table 1).

If further reduction of the contaminant level of the gas is required, an amine stripper can be used. As the solvent circulation rate and required reboiler energy are directly proportional to the contaminant level, the energy consumption of the amine treater after CRS is reduced by a factor 8 . The absorber is only slightly smaller due to the somewhat reduced gas flow, but all equipment after the absorber used to regenerate the amine reduces by a factor 8. Based on data for amine treatment from Alami (2010) and Kashani et al. (2010), Table 1 gives an overview of the energy consumption and size for amine treatment and CRS + amine treatment. The size of the combined installation is almost halved and the energy consumption is reduced by more than a factor 3 .

In the case of the Arabiyah and Hasbah gas fields, mixing the produced gas with gas of higher quality (Alami 2010) to reduce the level of inert $\mathrm{N}_{2}$ is proposed. In that case, the reduction of the $\mathrm{H}_{2} \mathrm{~S}$ level might be sufficient to eliminate the need for further (amine) treatment altogether. CRS as stand alone sweeting process would then become an option. An impression for the scale of the installation is given in Fig. 7.

In CRS, the acid is produced as a liquid. An alternative to producing elementary sulphur is to re-inject the acid underground. Transport of the concentrated liquid to sites
Fig. 5 Flow diagram of the two-stage separation process for a sour natural gas field

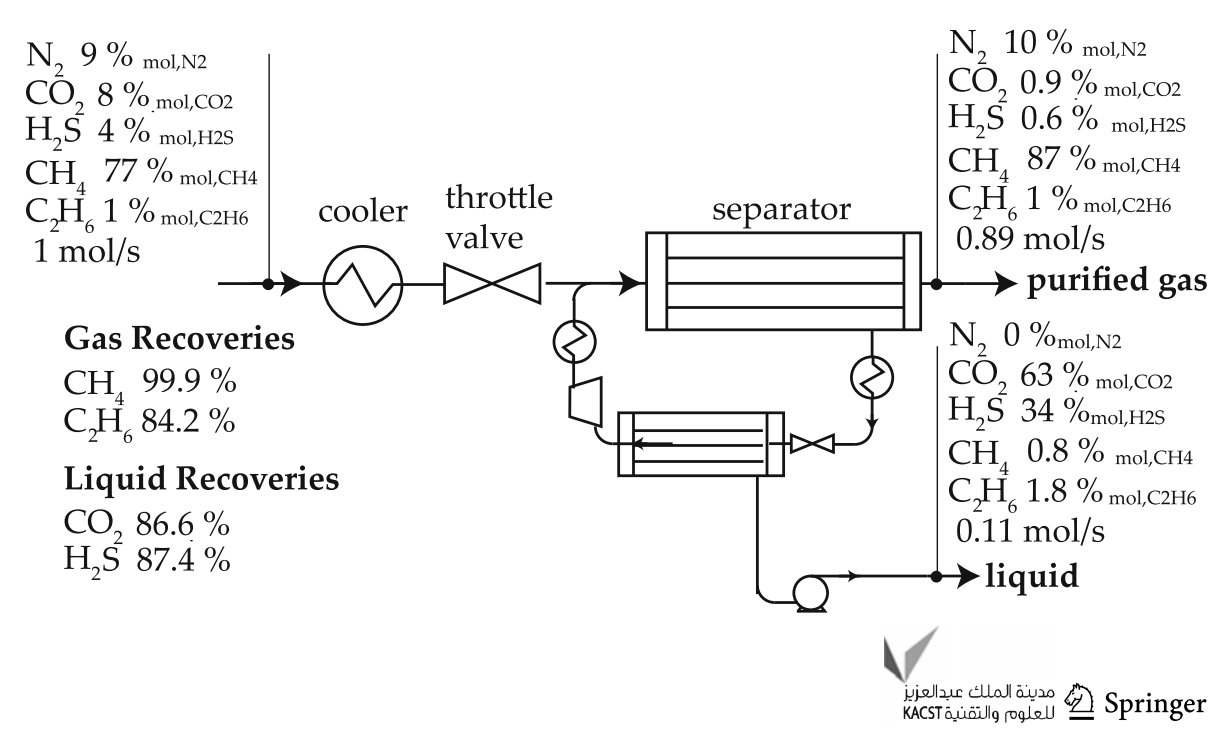



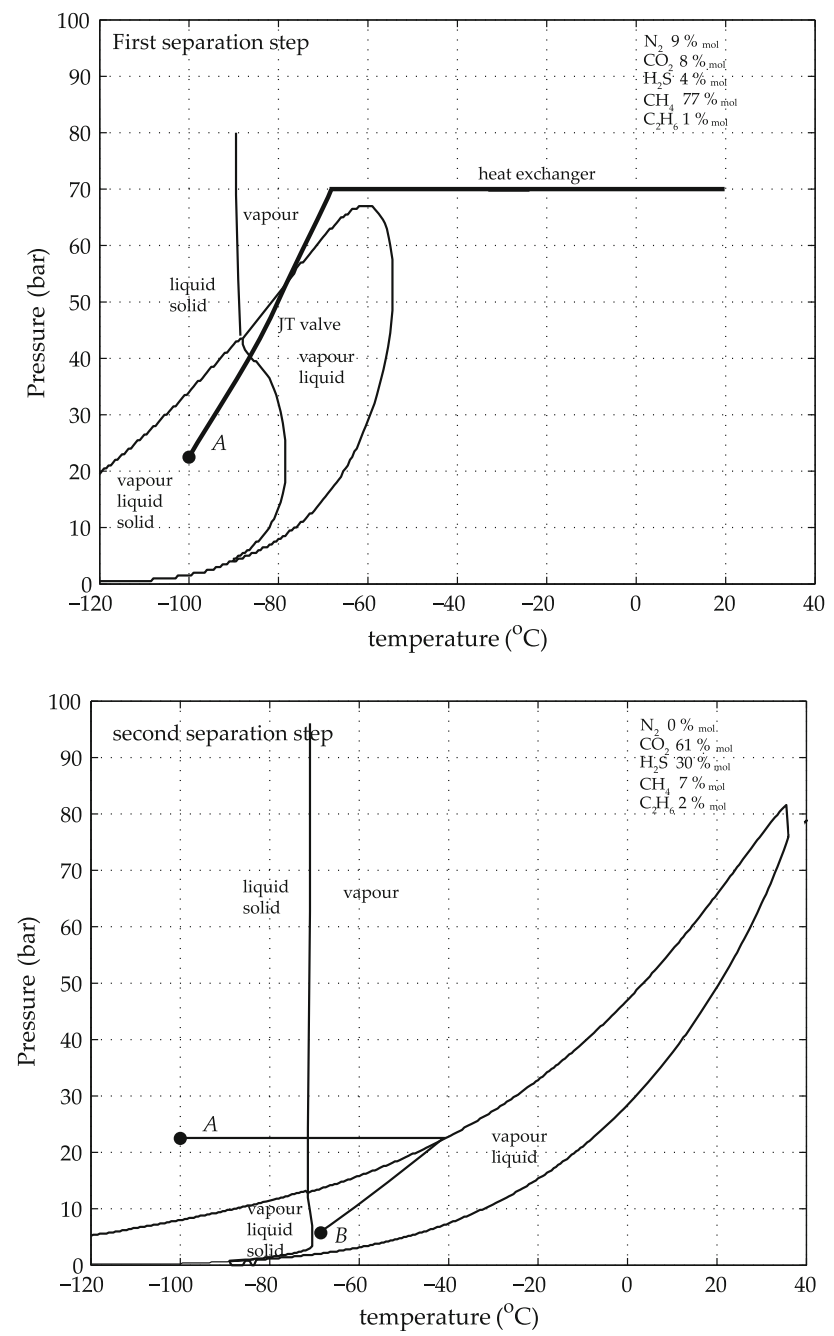

Fig. 6 P-T diagrams of the mixtures in the first and second separation step

Table 1 Energy consumption and size estimation between amine treatment and amine + CRS

\begin{tabular}{lccc}
\hline \multicolumn{1}{c}{ Amine } & CRS + Amine \\
\hline Energy consumption (\% of incoming heating value) & \\
Reboiler & 3.1 & & 0.37 \\
Cooler & 0.6 & 0.03 & 0.07 \\
Pumps & 0.2 & & 0.03 \\
Compressor & & 0.07 & \\
Hydrocarbons in liquid & & 0.54 & \\
Total & 3.8 & 0.64 & $+0.47=1.1$ \\
Volume estimate (m ${ }^{3}$ per $1 \mathrm{~kg}^{-1}$ feed gas) & \\
Absorber & 1.15 & & 1.01 \\
Stripper & 0.95 & & 0.10 \\
Cold box & & 0.04 & \\
RPS & & 0.02 & \\
Total & 2.05 & 0.06 & $+1.11=1.17$ \\
\hline
\end{tabular}

\section{cold box}

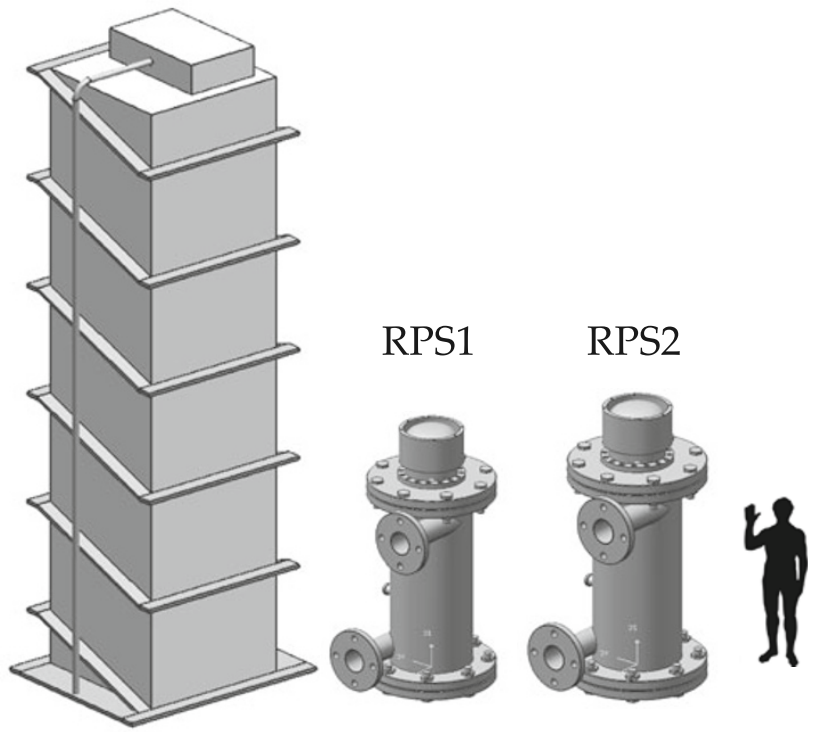

Fig. 7 Conceptual design of a $665 \mathrm{MMscf} /$ day CRS installation (on scale)

elsewhere is also an option, eliminating the need for an on-site Claus plant.

\section{Discussion}

Condensed rotational separation is a process of bulk separation based on flash evaporation or pressure distillation. The rotational particle separator provides the means to separate the micron-sized droplets formed after the flash. When applied to sour gas fields, the concentration of contaminants can be lowered to a level that allows economic application of conventional physical and/or chemical absorption processes. CRS is capable of processing sour gases with a wide range of $\mathrm{CO}_{2}$ and $\mathrm{H}_{2} \mathrm{~S}$ compositions. The benefits of using CRS becomes more distinguished with increasing sour gas content. But even for moderately contaminated fields where conventional methods are considered for gas sweetening, CRS can significantly lower the operational and capital costs as is shown for the Arabiyah and Hasbah gas fields in Saudi Arabia.

The methane produced from the CRS process can meet high purity specifications, i.e. as feedstock for LNG production. The sour components of the gas are removed as a liquid. This allows for economic reinjection for geosequestration or provides injectant for enhanced oil recovery.

Condensed rotational separation offers major benefits in capital and operating costs compared to amine treatment. Capital costs savings are a result of CRS being a simpler process with fewer process steps (Fig. 5). The equipment needed is less and smaller (Fig. 7). CRS has a higher 
process efficiency and uses less supplemental fuel (Table 1). Bulk sweetening by CRS can also provide a method to increase the total throughput of an existing treatment plant without the need for adding additional sulphur recovery capacity.

As proof of principle the first step of the CRS process was realized at lab scale and operated with $\mathrm{CH}_{4}-\mathrm{CO}_{2}$ mixtures in the vapour-liquid region (Willems et al. 2010; Bansal et al. 2011; Bansal 2012). The prediction tools for methane recovery and droplet size were confirmed. Work in progress is extending the experiments into the vapourliquid-solid regime with multi component mixtures.

Low temperature or Joule-Thomson expansion processes have been used in gas processing plants since the 1960s (Gas Processors Suppliers Association 2004). Except for the rotational particle separator, the equipment needed for the CRS process can be purchased as proven technology. The RPS has been demonstrated in an atmospheric test rig with water and air as working fluids (Willems et al. 2010b). The test setup approximately models a $24 \mathrm{~m} 3 / \mathrm{s}$ (80 MMscf/d) equivalent installation on a natural gas well. A prototype that can operate under pressure is constructed in preparation for a field test.

The notion that micron-sized droplets are formed in any separation process involving a phase change, led to the investigation of the technology for other area's of application: removal of condensate from natural gas, removal of $\mathrm{CO}_{2}$ from coal gasification producer gas and removal of $\mathrm{CO}_{2}$ from coal combustion gas. These applications are shortly discussed.

Natural gas condensate is a low-density mixture of hydrocarbon liquids that are present as gaseous components in the raw natural gas produced from many natural gas fields. The incoming gas is cooled to below the dewpoint of the hydrocarbons of the feedstock pressure. In the separator, a good part of the hydrocarbons is removed. Subsequently, the gas condensate is throttled to a low pressure separator. The reduction in pressure across the throttle valve causes the condensate to undergo a partial vaporization where even more hydrocarbons can be removed. The RPS separation technology as described above can directly be applied to this process with as compelling advantages: higher separation efficiency, smaller installation and lower energy usage.

Application of CRS to conventional coal combustion power plants shows that large improvements can be made in separation efficiency when combined with a relatively small oxygen enrichment of the air. The energy costs of separation are below those of existing carbon capture technologies, although low energy oxygen enrichment technologies need to be found. The key factor of the attractiveness of this technology is finding cheap methods for slight oxygen enrichment (Benthum et al. 2011).

\section{Conclusions}

The capability of collecting micron-sized droplets from gas streams by the rotational particle separator enables the use of pressure distillation as a compact alternative to fractional distillation. This, in turn, allows new process designs for:

1 upgrading sour gas fields

2 collecting valuable condensate from wet gas

$3 \mathrm{CO}_{2}$ removal in coal conversion.

Condensed rotational separation in the vapour-solidsliquid region and in the vapour solids region can be used as a bulk sweetening process prior to conventional techniques. Combination of CRS with conventional separation techniques can decrease both size and energy cost.

Operation of CRS within the vapor-liquid-solid region shows great potential for improvement of yields and purities. In the gas sweetening of light contaminated gas fields, CRS has the potential to produce sweetened natural gas at (almost) pipeline specifications, with a $\mathrm{CO}_{2}$ and $\mathrm{H}_{2} \mathrm{~S}$ content $<1 \%$ in the sweetened gas, while methane content in the waste is small, $<1 \%$.

The key elements of condensed rotational separation have been demonstrated at lab scale and semi-industrial scale. The next step is to start field tests of the RPS and perform a more detailed analysis of the CRS process and the other equipment.

Acknowledgments The authors wish to thank prof. Michael Golombok for fruitfull discussions. Romico Hold is acknowledged for access to proprietary knowledge regarding the RPS and related processes.

Open Access This article is distributed under the terms of the Creative Commons Attribution License which permits any use, distribution and reproduction in any medium, provided the original author(s) and source are credited.

\section{References}

Alami IA (2010) Wasit gas plant: new sour gas developments in Saudi Arabia. In: 6th Sour Oil and Gas Advanced Technology conference, Abu Dhabi, March 30-April 1, 2010

Austrheim A (2006) Experimental characterization of high-pressure natural gas scrubbers. PhD thesis, University of Bergen, Norway

Bansal GD (2012) Condensing $\mathrm{CO}_{2}$ droplets. PhD thesis, Eindhoven University of Technology

Bansal G, Golombok M, Brouwers JJH, Tesselaar G (2011) $\mathrm{CO}_{2}$ droplets condensed from natural gas. I\&EC research. 10.1021/ ie1012689. published online January 31

Bejan A (1997) Advanced engineering thermodynamics, 2nd edn. Wiley, New York

Burgers WFJ, Northrop PS, Khesgi HS, Valencia JA (2011) Worldwide development potential for sour gas. Energy Procedia 4:2178-2184

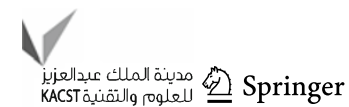


Clodic D, El Hitti R, Younes M, Bill A, Casier F (2005) $\mathrm{CO}_{2}$ capture by anti-sublimation. Thermo-economic process evaluation. In: National conference on carbon sequestration, DOE, Washington

Brouwers JJH, Kemenade HP van, Kroes JP (2012) Rotational particle separator: an efficient method to separate micron-sized droplets and particles. Filtration 12(1):49

Clift R (1997) Inertial separators: basic principles. In: Seville JPK (ed) Gas cleaning in demanding applications. Blackie, London

de Best CJJM, van Kemenade HP, Brunner T, Obernberger I (2008) Particulate emission reduction in small-scale biomass combustion plants by a condensing heat exchanger. Energy Fuels 22(1):587-597

Gas Processors Suppliers Association (2004) Engineering data book, 12th edn

Hart A, Gnanedran N (2009) Cryogenic $\mathrm{CO}_{2}$ capture in natural gas. Energy Procedia 1(1):697-706

Hinds WC (1999) Aerosol technology. John Wiley \& Sons, New York

Kashani N, Fernandez R, Giesen V, Katz T (2010) The biggest challange in sour gas: acid removal technologies! In: 6th Sour Oil and Gas Advanced Technology conference, Abu Dhabi, March 30-April 1, 2010

Kidnay WA, Parrish WR (2006) Fundamentals of natural gas processing. Taylor and Francis, Boca Raton

Linde AG Catalogue entitled Rohrbündel-Wärmeaustaucher. Linde A.G., Werksgruppe, TVT, Munich, Germany

Mougin P, Renaud X, Elbaz G (2008) Operational validation of the Sprex process for bulk $\mathrm{H}_{2} \mathrm{~S}$ and mercaptans removal. In: The gas industry: current \& future (6), 17-19 IFP, Rueil-Malmaison, France

Neeraas BA, Fredheim AO, Aunan B (2004a) Experimental shell side heat transfer and pressure drop in gas flow for spiral-wound LNG heat exchanger. Int J Heat Mass Transf 47:353-361

Neeraas BA, Fredheim AO, Aunan B (2004b) Experimental data and model for heat transfer, in liquid falling film flow on shell side, for spiral-wound LNG heat exchanger. Int J Heat Mass Transf 47:3565-3572

Olajire AA (2010) $\mathrm{CO}_{2}$ capture and separation technologies for endof-pipe applications. A review. Energy 35(6):2610-2628

Reid RC (1984) The properties of gases and liquids. McGraw-Hill, New York

Schaber K, Körber J, Ofenloch O, Ehrig R, Deuflhard P (2002) Aerosol formation in gas-liquid contact devices-nucleation, growth and particle dynamics. Chem Eng Sci (57):4345-4356

Schinkelshoek P, Epsom HD (2008) Supersonic gas conditioningcommercialisation of twister technology. In: 87th GPA annual convention, Grapevine, USA, March 2-5, 2008

Schweitzer PA (1996) Corrosion engineering handbook. Marcel Dekker, New York

Svarovsky L (1984) Hydrocyclones. Holt, London

Strauss W (1975) Industrial gas cleaning. 2nd edn. Pergamon, Oxford

van Benthum RJ, van Kemenade HP, Brouwers JJH, Golombok M (2011) Condensed rotational separation of $\mathrm{CO}_{2}$. Appl Energy. doi:10.1016/j.apenergy.2011.12.025

van Wissen RJE (2006) Centrifugal separation for cleaning well gas streams: from concept to prototype. $\mathrm{PhD}$ thesis, Eindhoven University of Technology, The Netherlands

VDI-Wärmeatlas (2006) 10th edn. VDI-Verlag, Düsseldorf, ISBN: 978-3-540-25504-8

Willems GP (2009) Condensed rotational cleaning of natural gas. $\mathrm{PhD}$ thesis, Eindhoven University of Technology

Willems GP, Kroes JP, Golombok M, Esch BPM van, Kemenade HP van, Brouwers JJH (2010) Performance of a novel rotating gasliquid separator. J Fluid Eng 132(3):031301

Willems GP, Golombok M, Tesselaar G, Brouwers JJH (2010) Condensed rotational separation of $\mathrm{CO}_{2}$ from natural gas. AIChE J 56(1):156 